\title{
Evaluation of risk factors associated with stroke
}

\author{
MS Ahamed ${ }^{1}$, MMH Chowdhury ${ }^{2}$, PK Chowdhury ${ }^{3}$, MA Hossain ${ }^{4}$
}

\begin{abstract}
Stroke is the leading cause of adult disability and is the third commonest cause of death worldwide. It is a major cause of morbidity and mortality with disability and social dependence throughout the world. An observational, descriptive, cross-sectional study was conducted among purposively selected one hundred and seventy patients with stroke admitted at Kushtia Medical College Hospital, Kushtia from July 2014 to June 2015 to evaluate the different modifiable risk factors and their frequency among stroke patients. Stroke patients with more than 24 hours duration presenting within seven days of onset irrespective of age, sex and demographic profile were included in the study. After obtaining informed consent, patients who fulfilled the inclusion criteria were thoroughly evaluated for the presence of risk factors. Data were collected by investigators themselves. Out of 170 confirmed cases of stroke, 89 (52.35\%) were male, while 81 (47.65\%) were female. Mean age of the patients was 63.74 years with a standard deviation of 16.5 years. The highest incidence $43(24.70 \%)$ of stroke was in the age group of 60 to 69 years. As many as 122 (71.76\%) had ischaemic stroke, while 48 (28.24\%) had haemorrhagic stroke. At least 119 (70.00\%) cases were hypertensive. 41 (24.11\%) cases had diabetes mellitus, 31 (18.23\%) had hyperlipidaemia, 52 (30.6\%) were smokers and 25 (14.70\%) had past history of stroke. Among 81 female patients, $66(81.48 \%)$ were in post-menopausal, whereas 15 (18.52\%) were in the reproductive age group. Of them 5 (33.33\%) had history of taking oral hormonal contraceptive. Hypertension, smoking, diabetes mellitus and hyperlipidaemia are the identified modifiable risk factors associated with stroke.
\end{abstract}

\section{Introduction}

Stroke is defined as rapidly developing symptoms and/or signs of focal, and at times global loss of cerebral function lasting for twenty four hours or more or earlier death with no apparent cause other than that of vascular origin. ${ }^{1}$ Traditionally, the term stroke has been used to include episodes of focal brain dysfunction due to focal ischaemia or haemorrhage. At present, it is the third leading cause of death worldwide, surpassed only by the heart disease and cancer. ${ }^{2}$ The clinical manifestations of stroke are highly variable because of the complex anatomy of the brain and its vasculature. Stroke can be broadly classified into ischaemic and haemorrhagic ones. Ischaemic stroke occurs due to loss of blood to part of the brain initiating ischaemic cascade due to free radical production and damage to the endothelial lining. Haemorrhage usually results from a rupture of a blood vessel within the brain parenchyma but may also occur in a patient with Subarachnoid Haemorrhage (SAH) if the artery ruptures into the brain substance as well as into the sub arachnoid space. Generally, the haemorrhage is very sudden in onset. However,
Bang Med J Khulna 2015; 48 : 24-27

with cerebral infarction it has gradual onset and is usually progressive over a period of day or so until it reached its peak. ${ }^{3}$ For the diagnosis of stroke, detailed history and thorough clinical examinations are mandatory followed by computerized tomography (CT) scan of the brain for confirmation. 4 CT Scan is a simple, non invasive and accurate investigation in distinguishing cerebral infarct from haemorrhage and more preferable to Magnetic Resonance Imaging (MRI) in the acute stage.

Exposure to multiple risk factors, modifiable ones, for several years play important role for the development of stroke, so much so that even a low socio economic status is also a risk factor but the most important ones are hypertension, diabetes mellitus, smoking and hyperlipidaemia. ${ }^{5}$ Morbidity and mortality from stroke can be reduced significantly by timely identification and correction of modifiable risk factors. 6 About $20.00 \%$ of the stroke survivors requiring institutional care after

1. Md Saad Ahamed M.phil, Associate Professor, Department of Radiology \& Imaging, Kushtia Medical College, Kushtia.

2. Mir Mahfuzul Hoque Chowdhury MD, Associate Professor, Department of medicine, Kushtia Medical College, Kushtia.

3. Poritosh Kumar Chowdhury DTCD, Associate Professor, Dept. of Respiratory Medicine, Khulna Medical College.

4. Md Altaf Hossain MD, Professor, Dept. of Radiology \& Imaging, Rangpur Medical College, Rangpur. 
three months and $15 \%$ to $30 \%$ being permanently disabled. 7 Identifying the risk factors and modifying them remain the most important means of reducing stroke incidence. This study was undertaken to determine the frequency of different modifiable risk factors among stroke patients admitted to a tertiary care hospital.

\section{Materials and methods}

This observational, descriptive, cross sectional study was conducted among purposively selected one hundred and seventy (170) patients with stroke, ischaemic or haemorrhagic, admitted in medicine unit of Kushtia Medical College Hospital Kushtia over a period of one year from July 2014 to June 2015. Stroke patients with more than twenty four hours duration presenting within seven days of onset, irrespective of age, sex and demographic profile, were included in the study. Patient with focal neuronal deficit or stroke like symptoms secondary to space occupying lesions, meningitis, encephalitis, blood dyscrasias, trauma and multiple sclerosis were not included in the study. Stroke was initially diagnosed on clinical ground and confirmed on CT Scan of brain. CT Scan of brain was carried out at one renowned private diagnostic centre, Kushtia

After obtaining informed consent, patients who fulfilled the inclusion criteria were thoroughly evaluated for the presence of risk factors. A detailed history followed by a thorough clinical examination was carried out before conducting the investigations. All the findings were recorded in Case Record Form (CRF). Background data like age, sex, residence and occupation were recorded. Moreover, history of hypertension, diabetes mellitus, smoking, and Transient Ischacmic Attack (TIA), use of anti diabetic or antihypertensive drugs and oral contraceptives in the females of reproductive age including previous history of stroke, myocardial infarction, angina, atrial fibrillation, and family history of stroke were sought. Investigations like blood glucose, lipid profile and complete blood count were carried out.

Hypertension was defined operationally as a past medical history of hypertension, patients taking anti-hypertensive therapy or systolic blood pressure at/over $140 \mathrm{~mm}$ of $\mathrm{Hg}$ and diastolic blood pressure at/over $90 \mathrm{~mm}$ of $\mathrm{Hg}$ on two separate occasions, Diabetes mellitus was diagnosed as a past medical history of diabetes mellitus or sustained blood sugar elevation (fasting blood sugar $7.0 \mathrm{mmol} / 1$ or above or blood sugar 02 hours after glucose load $11.1 \mathrm{mmol} / 1$ ), hyperlipidaemia was defined as cholesterol excess of $52 \mathrm{mmol} / 1$, triglycerides of more than 1.7 $\mathrm{mmol} / \mathrm{I}$ (fasting) LDL of more than $130 \mathrm{mg}$ per decilitre and HDL of less than $40 \mathrm{mg}$ per decilitre in man and $50 \mathrm{mg}$ per decilitre in woman irrespective of whether the patients were taking lipid lowering agent or not. Smoker was defined as a person who smoked at least one cigarette or biri per day for the preceding three months or more or had tobacco in any form. Data were collected by investigators themselves. After completing each case record form, it was checked for completeness, consistency and relevancy. Data were analyzed by using master sheet and scientific calculator.

\section{Result}

An observational, descriptive, cross-sectional study was conducted among patients with stroke admitted at Kushtia Medical College Hospital, Kushtia from July 2014 to June 2015 with a view to identify the modifiable risk factors of stroke with a sample size of one hundred and seventy. Among 170 confirmed cases of stroke, 89 (52.35\%) were male, while 81 were $(47.65 \%)$ female with a male female ratio of 1.09:1.00. Among 81 female patients, 66(81.48\%) were in post menopausal, whereas $15(18.52 \%)$ were in the respective age group. Highest age was 106 years and lowest age was 22 years with a range of 84 years. Mean age of the patients was 63.74 years with a standard deviation of 16.50 years. The highest incidence of stroke was in the age group of 60-69 years which comprised of $42(24.70 \%)$ of total patients (Table 1).

Table I

Age and sex distribution of patients with stroke

\begin{tabular}{lccc}
\hline \multirow{2}{*}{ Age in Years } & \multicolumn{3}{c}{ Sex } \\
& Male & Female & \\
& & & \\
$20-29$ & 1 & 3 & $4(2.35)$ \\
$30-39$ & 4 & 5 & $9(5.29)$ \\
$40-49$ & 6 & 13 & $19(11.17)$ \\
$50-59$ & 17 & 15 & $32(18.82)$ \\
$60-69$ & 23 & 19 & $42(24.70)$ \\
$70-79$ & 24 & 17 & $41(24.11)$ \\
$80-89$ & 10 & 5 & $15(8.82)$ \\
$90-99$ & 2 & 1 & $3(1.76)$ \\
$100-109$ & 2 & 3 & $5(2.940)$ \\
Total & $89(52.35 \%)$ & $81(47.65 \%)$ & $170(100)$ \\
\hline
\end{tabular}

* mean=63.74 years; $\mathrm{SD}=16.50$ years

Out of 170 patients, $122(71.76 \%)$ had ischaemic stroke, while $48(28.24 \%)$ had haemorhagic stroke. Of 122 ischaemic stroke patient, 67 (39.41\%) were males, while 55 (32.35\%) were females. Out of 170 patients, $48(28.24 \%)$ patients had haemorrhagic stroke with 22 (12.94\%) males and 26 (15.30\%) females (Table-II) 
Table II

Sex wise CT Scan Findings

\begin{tabular}{lccc}
\hline \multicolumn{4}{c}{ Sex } \\
CT Findings & Male(\%) & Female(\%) & Total (\%) \\
\hline Infarction & $67(39.41)$ & $55(32.35)$ & $122(71.76)$ \\
Haemorrhage, & $22(12.94)$ & $26(15.30)$ & $48(28.24)$ \\
Total & $89(52.35)$ & $81(47.65)$ & $170(100)$ \\
\hline
\end{tabular}

Out of 170 stroke patients, $119(70.00 \%)$ were hypertensive Of them, 60 (35.29\%) were males, whereas $59(34.71 \%)$ were females with a male female ratio of 1 : 0.98 . Of 170 patients, 122 $(71.76 \%)$ had cerebral infarction. Out of 122 cases of cerebral infarction, $82 \quad(67.21 \%)$ were hypertensive, while 48 cases were haemorrhagic stroke, $37(77.08 \%)$ were hypertensive. Of 170 stroke, $41(24.11 \%)$ had diabetes mellitus, while 31 $(18.23 \%)$ had hyperlipidaemia, $52(30.58 \%)$ were smokers, and $25(14.70 \%)$ had past history of stroke: (Fig-1). At least 26 (15.29\%) had both hypertension and diabetes mellitus, of which 11 $(42.31 \%)$ were males and $15(57.69 \%)$ were females. Out of 170 cases, $15(8.82 \%)$ females were in the reproductive age. Of them 5 (33.33\%) had history of taking oral hormonal contraceptive.

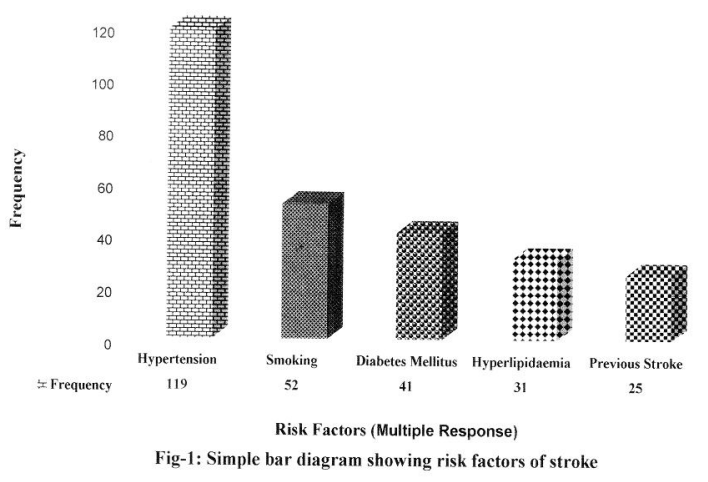

\section{Discussion}

Stroke is a leading cause of mortality and morbidity in both developed as well as developing countries. Stroke is a common medical emergency. About one fifth of patients with an acute stroke die within a month of the event, and at least half of those who survive are left with physical disability. 8

This descriptive, cross sectional study was conducted among 170 patients of stroke with the aim to determine the frequency of modifiable risk factors in patients admitted to Kushtia Medical College Hospital Kushtia, Bangladesh, with a view to reduce stroke related morbidity and mortality.
Out of 170 stroke patients, $119(70.00 \%)$ were hypertensive. Of them, 60 (50.42\%) were males, whereas 59 (49.58\%) were females with a male female ratio of 1: 0.98 . This findings correlate with that of other studies where hypertension was observed in $73.00 \%, 68.00 \%$ and $69.10 \%$ cases. $9,10,11$ Moreover, the findings are more or less consistent with findings of the Studies where the frequencies of hypertension were $50.00 \%$, $60.00 \%, 59.30 \%, 65.80 \%$ and $75.00 \% .12-17$ In this study, $67.21 \%$ patients of cerebral infarction and $77.08 \%$ patients of haemorrhagic stroke were hypertensive. Hypertension is the most prevalent and powerful modifiable risk factor for both ischaemic and haemorrhagic stroke irrespective of geographic region and ethnic group. ${ }^{18}$ Persons with hypertension are about 3 or 4 times more likely to have a stroke. ${ }^{19}$ Risk of stroke can be reduced by at least $38 \%$ with control of hypertension. ${ }^{14}$

Diabetes mellitus is another important modifiable risk factor for the stroke patient. One population based survey on stroke prevalence revealed that there is a $7 \%$ increased risk of stroke or transient ischaemic attack in patient with diabetes.20 Among 170 stroke patients in this study, $41(24.11 \%)$ had diabetes mellitus. This is in line with the findings of the studies conducted by others where stroke patients were diabetic in $45.00 \%, 42.50 \%, \quad 33.40 \%, 26.00 \%, 25.00 \%$, $20.00 \%$ and $18.00 \%$ cases. $9,11-13,21-23$ The reason of this variation could not be explained.

Smoking appeared as second most common modifiable risk factor for stroke in our study. Out of total, 89 male patients were with stroke. Of them, 52 (58.43\%) were smokers. This finding has similarity with the findings of $44.40 \%$ and $53.00 \% 25$ smokers among stroke patients. 24

Hyperlipidaemia was observed in 31 (18 23\%) out of 170 stroke patients. This finding closely resembles with the findings of other studies where the frequencies of hyperlipidaemia. were $18.00 \%$ and $19.00 \% .12,26$ Twenty six (15.29\%) patients out of 170 stroke patients, in this study were having both hypertension and diabetes mellitus. The two risk factors often co-exit and increases the chances of stroke. ${ }^{23}$

\section{Conclusion}

Based on findings it can be concluded that hypertension, smoking, diabetes mellitus and hyperlipidaemia are the identified modifiable risk factors associated with stroke. Earlier detection, treatment and modification of these factors can reduce the incidence and prevent the development of stroke that need to create awareness for changing baneful life style as well as regular use of medication. 


\section{References}

1. Devenport R Dennis M. 'Neurological emergencies: Acute stroke. J 'Neurol Neurosurgery psychiatry 2000: 68: 277-88.

2. Donnan GA Fisher M. Macleod M, Davis SM. Stroke. Lancet 2008; 371: 1612-23.

3. Ahmed MM. Nasirullah M. Study of clinical presentation versus CT findings regarding the type of lesion in stroke. Pak J Neuro 2004; 10: 17-22.

4. Rehman SU, Khan MA. Clinical versus CT Scan diagnosis in stroke: a comparative study of 50 cases. J Ayub Med Coll, 2002; 14: 2-5.

5. Onuwuchewa A, Bellgam. H, Asekomeh G. Stroke at the university of port harconet teaching hospital, rivers state, Nigeria Trop doct 2009; 39: 150-2.

6. lqbal F, Hussain S, Hassan M. Hypertension, diabetes mellitus and hyperlipidaemia as risk foctors for stroke. Pak J Med Res 2003; 42: 17-22.

7. American Heart Association. Heart disease and stroke statistics 2004 update. Dallas, texas the association 2003

8. P. Langhore. Stroke Disease, In : Davidson's Principles \& Practices of Medicine, 22nd Edition, Brain RW. Nick RC, Stuart HR, Lan DP Editors, London, Churchil Livingstone, 2010 ; 1237.

9. Tairq M Muhammad SA, Shahid I, Naila K, Hira M. Evaluation of risk factors in ischaemic stroke Journal of Rawalpindi medical college 2013 ; 17 : 138-9

10. Omkar PB, Susmita C, Ksh GV. Clinico epidemiological study of acute ischaemic stroke in a tertiary hospital of northeastern state of India. International journal of biomedical and advance research 2013 ; 04: 661-5

11. Aly $Z$ Abbas K, Kazim SF, Taj F, Aziz F. Irfan A. Awareness of stroke risk factors sign and treatment in a Pakistani population. Journal of Pakistan medical association 2009 ; 59: 494-9.

12. Vohra EA Ahmed WU, Ali M. Etiology and prognostic factors of patient admitted for stroke $\mathrm{J}$ Pak Med Assoc 2000; 50 : 234-6.

13. Asghar K . Siddique A, Selim K. Frequency of risk factors in stroke patients admitted to DHQ teaching hospital D I. khan Gomal Journal of Medical sciences 2010; 2; 200-03.
14. Suhail AA, Muzaffor S Muntaz AS, Khalid S, M.Qasim R, Ghulum H B and M. Iqbal S. Stroke: Frequency of risk factors in patients admitted at Liaquat University Hospital Hyderabad / Jamshoro 2008; 151-6.

15. Khan SN, Vohra EA, Risk factors for stroke: a hospital based study.Pak J Med Sci 2007; 23: 1722 .

16. Marwat MA, Usman M, Hussain M, Stroke and its relationship to risk factors. Gomal Journal of Medical science 2009 ; 7: 17-21.

17. Jaffor TH . Blood pressure, diabetes and increased dietary salt associated with stroke results from a community based study in Pakistan $\mathrm{J}$ Hum Hypertens 2006, 20: 83-5.

18. Du X, Cruickshank K, McNamee R, Saraece M, Sourbatts J, Summers A, et al. Case control study of stroke and quality of hypertension control in North West England. BMJ 1997; 314:272-6.

19. Javed MA, Ahmed A, Sial MSH, Naheed T, Risk factor in stroke Pak J New ol 1998 ; 4: 55-8.

20 Kamal AK, Ifrat a, Murtaza M, Khan M, Rasheed A, Ali A. The burden of stroke and transient ischamic attack in Pakistan: A community based prevalence study BMC Neurol 2009; 9: 58.

21. Syed. NA, Khealani BA, Ali S, Hasan A, Akhtar N, Brohi H (2003): 1schaemic stroke subtypes in Pakistan : the Aga Khan University stroke Data Bank. J Pak Med Assoc 2003; 53 : 584-8.

22 Safer M, Tariq M, Ubaid R. Frequency of risk factors of cerebral infarction in stroke patient. A study of 100 patients in Naseer Teaching Hospital Peshawar 2008; 24:109-13.

23. Amjad Z, Rabida K, Usman K, Kausar T, Mohd N. Frequency of diabetes is a major risk factors in stroke patients at Hyatabad Medical Complex Peswar, 2011; 3 : 70-1.

24 Hassan SR, Ghouri ASK . Frequency of known risk factors in stroke and its outcome in patient admitted in Sindh Government Qatar Hospital Karachi, Pakistan J Med Sci 2007; 23.

25. Basharat RA, Yousaf M, Iqbal J, Khan MM. Frequency of known risk factors for stroke in poor patients admitted to Lahore General Hospital in 2000. Pak J Med Sci 2002, 18: 280-3.

26 Khan H, Afridi AK, Ashraf S, A Hospital based study on stratification of risk factors for stroke in Peshwar Pak J Med Sci 2006 ; 22: 304-7 